\title{
Research on Fault Diagnosis of a Certain Launching Vehicle Based on Multi Attribute Decision Making Theory
}

\author{
Sai Wang*, Jiping Cao, Rui Huang and Ning Lei \\ Department of Management, Rocket Engineering University of Xi'an 710025, Shanxi, China \\ ${ }^{*}$ Corresponding author
}

\begin{abstract}
A fault tree diagnosis method for chassis braking system of launching vehicle is proposed in this paper based on multiple attribute decision making theory. Aiming at the limitation of the traditional fault tree analysis method of poor accuracy and low efficiency, this paper uses TOPSIS method to determine the best diagnosis sequence of bottom events considering the failure probability, the cost for diagnosis and the correlation value of bottom events and raises the precision of the bottom event diagnosis. The fault diagnosis and verification of a certain type of launching vehicle chassis braking system show that the method can accurately diagnosis the fault and improve the diagnosis efficiency.
\end{abstract}

Keywords-launching vehicle; chassis braking system; multiple attribute decision making; fault tree analysis method; fault diagnosis

\section{INTRODUCTION}

Fault tree analysis[1] (Fault Tree Analysis, FTA) is a graphic deduction method, which uses the prior knowledge of failure rate and fault cause to diagnose the final fault of equipment, and builds fault tree by studying the causes of various phenomena continuously. On the basis of fault tree, we can diagnose the cause of failure based on the real-time dynamic data of the fault tree, and find out the cause of the failure, which is widely applied in the field of mechanical fault diagnosis. The research of fault diagnosis by fault tree analysis at home and abroad is mainly as follows: Li Tianke et al designed the fault diagnosis platform for the control equipment of missile launcher based on FTA[2]; Cao Ming ea tl used FTA to diagnose a certain type of seeker quickly and got high diagnostic efficiency[3]; Li Shuying and others studied the BDD algorithm and applied it to FTA, which solved the problem of "dimension explosion" in FTA[4]; Zhang Yan et al integrated Petri network and FT to design the fault diagnosis model of power system[5]; Yao Kairui and others have studied the fault of the electrical system of the missile launcher, and designed the fault diagnosis expert system based on FT[6]; Xiao Guangyuan and others applied fuzzy set theory to build the fault tree model of the storage and launch box, which overcomes the difficulty of obtaining the probability of failure occurrence to a certain extent7]; He Jiazhou used the method of qualitative analysis and quantitative phase separation to construct a fault tree of a radar launch system[8]; Dong Zewei ea tl integrated FTA and the expert experience to construct the fault diagnosis model of a helicopter weapon system[9]; Yao Chengyu and others overcomed the limitation of the two state hypothesis of fault tree search and realized the diagnosis method based on T-S fuzzy fault tree analysis[10]. Aiming at solve the limitation of FTA, the research above integrate Petri network, BDD, fuzzy theory, TOPSIS and expert system to provide a train of thought for the chassis fault diagnosis of launch vehicle. In the process of fault tree diagnosis, the traditional way decides the order of diagnosis according to the probability of bottom event, which is inefficient in the diagnosis of chassis of actual launch vehicle[11-12] while the structure is complex , the difficulty and cost of each component's "hypothesis verification" process are quite different,and the value of the associated values we can get is also different from each other. Therefore, in the process of bottom event diagnosis, we should take account of the diagnostic cost (including manpower, resources and funds) and the correlation value in addition to the probability of failure occurrence, and then extract the diagnostic order after synthesizing all kinds of information. In this paper, a multi attribute decision theory is used to diagnose the fault tree of the chassis of the launching vehicle and a fault tree diagnosis method based on the multi attribute decision theory is proposed. In the case of fault tree bottom event probability $\mathrm{P}$ known, we integrated the bottom events diagnosis cost $\mathrm{D}$ and correlation value $\mathrm{V}$ as the decision attribute, using TOPSIS method (Technique For Order Preference By Similarity To Ideal Solution, TOPSIS) to determine the sequence of diagnosis, which can improve the efficiency of fault diagnosis and save the resource.

\section{CONSTRUCtion OF FAUlT TREe}

In the chassis system of a certain type of launch vehicle, any malfunction of the brake subsystem directly affects the braking effect, including the launch vehicle can not quickly decelerate and stop when braking, , it is deviated from the normal running road when braking, it can not relieve the braking effect or slow the braking effect and so on. Any one of the above failures will pose a serious threat to the operation of the launch vehicle, reducing the service life of the launch vehicle, even causing equipment damage and casualties. This paper takes the failure of the chassis brake system of a certain type of launching vehicle as the top event and sets up the brake 
system fault tree by analyzing the fault mechanism and expert

experience, as shown in Figure 1.



FIGURE I. FAULT TREE OF BRAKE SYSTEM

Brake system failure is taken as the top event and the basic event is $S_{1}$ (The brake drum turns too fast), $S_{2}$ (Breaking and falling off of tension spring), $S_{3}$ (Adjusting bolt breaking), $S_{4}$ (Brake drum ablation), $S_{5}$ (Brake belt wear), $S_{6}$ (Improper clearance adjustment), $S_{7}$ (Force spring off), $S_{8}$ (Elasticity reduction), $S_{9}$ (Brake belt fracture), $S_{10}$ (Arc hole grinding deviation), $S_{11}$ (Loosening of linkage mechanism), $S_{12}$ (Joint pin bending over dirty).

\section{FAULT LOCATION OF BRAKE SYSTEM BASED ON FTA}

In the fault tree of the brake system, the bottom event represents the possible cause of the failure and the failure part. The traditional fault tree analysis method traverses the bottom events of the fault tree and carries out "hypothesis verification" for the fault components listed on each bottom event, eliminating or confirming them one by one until the location and cause of the fault are found.

In the specific "hypothesis verification" process, we can judge whether the meta component involved in the bottom event has a failure by using the external fault phenomena observed, or performance parameters of the components which can be measured by the sensors and instruments installed in the system, or using some chassis components diagnostic instruments or methods, or by changing the components and observe whether the system returns to normal. For example, the $S_{2}$ and $S_{7}$ bottom events of the brake fault tree can be excluded or confirmed by external observation. For the bottom event $S_{1}$, if the instrument measures the brake drum speed exceeding the threshold limit, the $S_{1}$ event can be confirmed. For the bottom event $S_{12}$, we need to replace or restore the connection pin to determine whether the system is restored to normal. But for some bottom events as $S_{8}$, it is necessary to perform performance testing in addition to the necessary disassembly and assembly. Besides, the hypothesis verification process of each bottom event is not only isolated from the current, but also provides evidence or information for the other "hypothesis verification" process of the bottom events. Take $S_{8}$ as an example, the performance test results of the force spring can provide strong evidence for judging the failure of the auxiliary spring. If the spring performance is intact, the bottom event $S_{7}$ and $S_{8}$ can be excluded together.

In the process of bottom event diagnosis, if we assume that the probability of occurrence of the bottom events is the same, then the FTA uses sequential diagnosis. That is to diagnose $S_{1}$ first, then diagnose $S_{2}$, and diagnose the others in turn until we find the location and reason of the fault, in which great blindness exits.

In the actual process, the probability of the failure of each bottom event is different. The manufacturer and building user accumulate the maintenance record of the launch vehicle for a long time to get the probability of the failure of each event. Table 1 shows the probability of occurrence of the fault tree bottom event of the brake system. 
TABLE I. PROBABILITY OF OCCURRENCE OF FAULT TREE BOTTOM EVENT IN THE BRAKE SYSTEM OF A CERTAIN LAUNCHING VEHICLE

\begin{tabular}{|l|c|c|c|c|c|c|c|c|c|c|c|c|}
\hline Bottom event & $S_{1}$ & $S_{2}$ & $S_{3}$ & $S_{4}$ & $S_{5}$ & $S_{6}$ & $S_{7}$ & $S_{8}$ & $S_{9}$ & $S_{10}$ & $S_{11}$ & $S_{12}$ \\
\hline $\begin{array}{l}\text { Probability of } \\
\text { occurrence }\end{array}$ & 0.6 & 0.6 & 0.85 & 0.25 & 0.2 & 0.15 & 0.7 & 0.5 & 0.65 & 0.75 & 0.65 & 0.1 \\
\hline
\end{tabular}

The diagnosis sequence of the bottom event of the brake system failure is $S_{3}, S_{10}, S_{7}, S_{9}, S_{11}, S_{1}, S_{2}$, $S_{8}, S_{4}, S_{5}, S_{6}, S_{12}$ according to the probability of occurrence. But when the fault search order is determined in practice, some other factors ${ }^{[11]}$ must be considered to determine the successively of the diagnosis of the bottom event. The first is the cost of diagnosis, including the maintenance technicians, the tools and equipment used, the spare parts and expenditure invested, and so on., which is different from each component diagnosis ("hypothetical verification" process). Some failures can be found by external observation, such as pull spring break off, or force spring off, some faults must be determined by means of instruments or sensors, such as the overspeed of the brake drum, the elasticity of the support spring, etc, while some of the fault elements must be dismantled and replaced to confirm the failure. Besides, the detection difficulty of the brake system components is different either. For example, the inspection work includes observation, disassembly and replacement, some components disassembly will also affect the life and performance of the components.

The second factor to consider is the diagnostic relevance value contained in the sequence of diagnosis as mentioned before. The "hypothetical verification" process for each bottom event is not isolated, and may provide evidence or information for the diagnosis process of the subsequent bottom events. In the case of different bottom events, the amount of information is different, so the value of the associated value is different. After giving the above considerations, we use the multiple attribute decision making theory to determine the optimal diagnosis sequence of the bottom events of fault tree, which makes the process of fault diagnosis and location more rapid, effective and economical.

\section{Fault Tree Diagnosis Method Based on Multi ATTRIBUTE DECISION THEORY}

\section{A. Decision Matrix and Attribute Value Normalization}

MA represents a multi attribute decision making problem and $X=\left\{x_{1}, \ldots, x_{m}\right\}$ is an optional set of decisions. The $n$ attribute values of the diagnostic scheme $x_{i}$ are expressed as $Y_{i}=\left(y_{i 1}, \ldots, y_{i n}\right)$, in which the $j$ attribute value of the $i$ diagnosis scheme is recorded as $y_{i j}$. When the target function is $f_{j}, y_{i j}=f_{j}\left(x_{i}\right), i=1, \ldots, m, j=1, \ldots, n$. The attribute values of each plan are listed as decision matrices, as shown in table 2 , in which the data are the basic information needed for decision diagnosis and data pretreatment and diagnosis sequence solution are based on them.
TABLE II. DECISION MATRIX

\begin{tabular}{|c|c|c|c|c|c|}
\hline Symbol & $y_{1}$ & $\ldots$ & $y_{i}$ & $\ldots$ & $y_{n}$ \\
\hline$x_{1}$ & $y_{11}$ & $\ldots$ & $y_{1 j}$ & $\ldots$ & $y_{1 n}$ \\
\hline$\ldots$ & $\ldots$ & $\ldots$ & $\ldots$ & $\ldots$ & $\ldots$ \\
\hline$x_{i}$ & $y_{i 1}$ & $\ldots$ & $y_{i j}$ & $\ldots$ & $y_{i n}$ \\
\hline$\ldots$ & $\ldots$ & $\ldots$ & $\ldots$ & $\ldots$ & $\ldots$ \\
\hline$x_{m}$ & $y_{m 1}$ & $\ldots$ & $y_{m j}$ & $\ldots$ & $y_{m n}$ \\
\hline
\end{tabular}

Before making decision by using multiple attribute values, the data of each attribute needs to be normalized. The quantization specification is linear. It can make the sum of squares of all programs in the same attribute value change to 1 , which is widely used in the calculation of Euclidean distance between each scheme and a virtual solution. Therefore, both the cost and the benefit attributes are normalized by vector, as shown in formula (1):

$$
z_{i j}=y_{i j} / \sqrt{\sum_{i=1}^{m} y_{i j}^{2}}
$$

\section{B. Determination of Attribute Weighted Value}

The importance of attribute is quantified by weight value. It is difficult to determine the weight if the attribute is so many. For example, in decision makers, the importance of attribute A is 4 times that of $\mathrm{B}$, and the importance of attribute $\mathrm{B}$ is 2 times that of C. But most of the time, decision makers do not think the importance of attribute $\mathrm{A}$ is 8 times that of $\mathrm{C}$. At this point, it is necessary to aggregate the result of the contrast between attributes and determine the weight of the attribute group by a specific method. In this paper, the least square method is used to deal with it.

Taking $n$ attributes as an example, the decision maker compares the importance in pairs and compares $C_{n}^{2}=\frac{1}{2} n(n-1)$ times.

$a_{i j}$ is used to represent the relative importance of the $i$ attribute to the $j$ attribute, and it is approximated as the ratio of the weight $w_{i}$ of the attribute $i$ to the weight $w_{j}$ of the attribute $j$, as $a \approx w_{i} / w_{j}$. Matrix $A$ is used to represent the results of 5 attributes in pairs, as shown in formula (2). 


$$
A=\left[\begin{array}{cccc}
a_{11} & a_{12} & \cdots & a_{1 n} \\
a_{21} & a_{22} & \cdots & a_{2 n} \\
\vdots & \vdots & & \vdots \\
a_{n 1} & a_{n 2} & \cdots & a_{n n}
\end{array}\right] \approx\left[\begin{array}{cccc}
w_{1} / w_{1} & w_{1} / w_{2} & \cdots & w_{1} / w_{n} \\
w_{2} / w_{1} & w_{2} / w_{2} & \cdots & w_{2} / w_{n} \\
\vdots & \vdots & & \vdots \\
w_{n} / w_{1} & w_{n} / w_{2} & \cdots & w_{n} / w_{n}
\end{array}\right]
$$

If the decision maker can accurately estimate $a_{i j}(i, j \in J)$, there is formula (3) and (4).

$$
a_{i j}=1 / a_{j i}, a_{i j}=a_{i k} \cdot a_{k j},(\forall i, j, k \in J)
$$

$$
\sum_{i=1}^{n} a_{i j}=\frac{\sum_{i=1}^{n} w_{i}}{w_{j}}
$$

When $\sum_{i=1}^{n} w_{i}=1$, there is formula (5).

$$
w_{j}=\frac{1}{\sum_{i=1}^{n} a_{i j}}
$$

If $a_{i j}$ can not be accurately estimated, the equal number in the upper form is changed to the approximate number, and the least square method is used to find the $\boldsymbol{w}$, that is, the solution of formula (6).

$$
\min \left\{\sum_{i=1}^{n} \sum_{j=1}^{n}\left(a_{i j} w_{j}-w_{i}\right)^{2}\right\}
$$

Restricted to the formula (7):

$$
\sum_{i=1}^{n} w_{i}=1, w_{i}>0(i=1,2, \cdots, n)
$$

The construction of Lagrange's function, as shown in formula (8):

$$
L=\sum_{i=1}^{n} \sum_{j=1}^{n}\left(a_{i j} w_{j}-w_{i}\right)^{2}+2 \lambda\left(\sum_{i} w_{i}-1\right)
$$

$n$ equations are obtained for the partial derivative of $w_{l}(l=1,2, \ldots, n)$, as shown in formula (9).

$$
\sum_{i=1}^{n}\left(a_{i l} w_{l}-w_{i}\right) a_{i l}-\sum_{j=1}^{n}\left(a_{l j} w_{j}-w_{l}\right)+\lambda=0, l=1,2, \cdots, n
$$

The formula (9) and $\sum_{i=1}^{n} w_{i}=1$ consist of $n+1$ equations, in which $n+1$ variables are consist with $w_{1}, w_{2}, \cdots, w_{n}$ and $\lambda$,to get the result $w=\left[w_{1}, w_{2}, \cdots, w_{n}\right]$.

\section{TOPSIS Sorting Method}

TOPSIS is a ranking method(technique for order preference by similarity to ideal solution)that approximates the ideal solution, which makes use of the ideal solution and negative ideal solution of multiple attribute problems to sort each plan in the solution set.

$X=\left\{x_{1}, x_{2}, \cdots, x_{m}\right\}$ is an alternative set of multiple attribute decision problems, and $Y=\left\{y_{1}, y_{2}, \cdots, y_{n}\right\}$ is an attribute vector to measure the quality of the scheme. The $n$ attribute values of each scheme $(i=1, \cdots, m)$ in $X$ can form a vector $Y_{i}=\left\{y_{i 1}, y_{i 2}, \cdots, y_{i n}\right\}$, which is set as the only point in the $n$ dimensional space that represents the $x_{i}$.

The best solution in $X$ is expressed with $x^{*}$, and most of the time $x^{*}$ does not exist, that is, it is the ideal solution and its attribute value is the best value in the decision matrix. In the same way, $x^{0}$ is used to express the worst scheme in $X$, that is, the negative ideal solution, and its attribute values are the most differential values.

Comparing the distances between $x_{i}$ and $x^{*}$ and between $x_{i}$ and $x^{0}$ in n-dimensional space, the best solution in $X$ is the least distance from the ideal solution and the maximum distance from the negative ideal solution. Accordingly, we can prioritize the order.

\section{Fault Tree Diagnosis of the Chassis of A CERTAin LAUNCH VEHICLE}

The application of multi attribute decision theory in fault tree diagnosis is carried out by taking the chassis brake system fault of a certain launching vehicle as an example. Figure 1 shows the fault tree and bottom event of the brake system.

The bottom event occurrence probability $\mathrm{P}$, the diagnostic cost $\mathrm{D}$ and the associated value $\mathrm{V}$ given by the statistical data provided by the manufacturers and the users are used as decision making properties, to form the decision matrix of fault tree for brake system with optimal diagnosis order, as shown in table 3. $i$ represents the bottom event sequence number, $j$ is the attribute number, and the occurrence probability $y_{1}$ ranges from 0 to 1 , the diagnostic cost $y_{2}$ ranges from 0 to 100 , the associated value $y_{3}$ ranges from 0 to 10 . 
TABLE III. DECISION MATRIX Y OF OPTIMAL DIAGNOSIS SEQUENCE OF FAULT TREE FOR BRAKE SYSTEM

\begin{tabular}{|c|c|c|c|}
\hline${ }_{i}^{j}$ & $y_{1}$ & $y_{2}$ & $y_{3}$ \\
\hline 1 & 0.6 & 1 & 3 \\
\hline 2 & 0.6 & 2 & 3 \\
\hline 3 & 0.85 & 20 & 8 \\
\hline 4 & 0.25 & 35 & 5 \\
\hline 5 & 0.2 & 40 & 4 \\
\hline 6 & 0.15 & 55 & 3 \\
\hline 7 & 0.7 & 8 & 3 \\
\hline 8 & 0.5 & 25 & 5 \\
\hline 9 & 0.65 & 80 & 3 \\
\hline 10 & 0.75 & 80 & 3 \\
\hline 11 & 0.65 & 10 & 4 \\
\hline 12 & 0.1 & 70 & 2 \\
\hline
\end{tabular}

A. Normalization Decision Matrix Calculation

Set $Y=\left\{y_{i j}\right\}$ as a multi attribute decision matrix and $Z=\left\{z_{i j}\right\}$ as a normalized multi attribute decision matrix, we can get the formula (10).

$$
z_{i j}=y_{i j} / \sqrt{\sum_{i=1}^{m} y_{i j}{ }^{2}}, i=1, \cdots, m ; j=1, \cdots, n
$$

The vector normalized decision matrix can be obtained after the attribute values in table 4 are processed with formula (10), whose attribute value is shown in table 4.

TABLE IV. VECTOR NORMALIZED DECISION MATRIX $Z$

\begin{tabular}{|c|l|l|l|}
\hline$i$ & $z_{1}$ & $z_{2}$ & $z_{3}$ \\
\hline 1 & 0.3109 & 0.0064 & 0.2100 \\
\hline 2 & 0.3109 & 0.0127 & 0.2100 \\
\hline 3 & 0.4404 & 0.1271 & 0.5601 \\
\hline 4 & 0.1295 & 0.2225 & 0.3501 \\
\hline 5 & 0.1036 & 0.2543 & 0.2801 \\
\hline 6 & 0.0777 & 0.3496 & 0.2100 \\
\hline 7 & 0.3627 & 0.0509 & 0.2100 \\
\hline 8 & 0.2591 & 0.1589 & 0.3501 \\
\hline 9 & 0.3368 & 0.5086 & 0.2100 \\
\hline 10 & 0.3886 & 0.5086 & 0.2100 \\
\hline 11 & 0.3368 & 0.0636 & 0.2801 \\
\hline 12 & 0.0518 & 0.4450 & 0.1400 \\
\hline
\end{tabular}

\section{B. Determine the Weight of Each Attribute}

As the weighted standard array $X=\left\{x_{i j}\right\}$ is formed, we assume that $\boldsymbol{w}=\left(w_{1}, w_{2}, \cdots, w_{n}\right)^{T}$ is given by the decision maker, and there is formula (11).

$$
x_{i j}=w_{j} \cdot z_{i j}, i=1, \cdots, m ; j=1, \cdots, n
$$

In this case, the domain experts can directly give the weight vector $\boldsymbol{w}$ according to the actual fault diagnosis of the chassis system. However, in the actual process, the experts do not give the right value of each attribute because of the complexity of the various factors. They often compare each attribute in pairs, while this comparison may be inaccurate or inconsistent. So we use the least square method to deal with it.

The matrix $A$ given by experts with comparison of each attribute in pairs is shown as follows.

$$
\begin{aligned}
& \begin{array}{lll}
P & D & V
\end{array}
\end{aligned}
$$

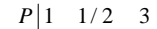

$$
\begin{aligned}
& A=D \mid \begin{array}{lll}
2 & 1 & 5
\end{array} \\
& \text { V } 1 / 3 \quad 1 / 5
\end{aligned}
$$

Using the least square method to solve the weight matrix, we can get $w=\left[\begin{array}{lll}0.3015 & 0.5859 & 0.1126\end{array}\right]$

The weighted matrix $Z^{\prime}$ can be obtained by using the formula (11) to calculate the weighted normalized decision matrix, as shown in table 5

TABLE V. WEIGHTED NORMALIZED DECISION MATRIX $Z^{\prime}$

\begin{tabular}{|c|l|l|l|}
\hline$i \quad j$ & $z_{1}^{\prime}$ & $z_{2}^{\prime}$ & $z_{3}^{\prime}$ \\
\hline 1 & 0.0937 & 0.0037 & 0.0237 \\
\hline 2 & 0.0937 & 0.0074 & 0.0237 \\
\hline 3 & 0.1328 & 0.0745 & 0.0631 \\
\hline 4 & 0.0391 & 0.1304 & 0.0394 \\
\hline 5 & 0.0312 & 0.1490 & 0.0315 \\
\hline 6 & 0.0234 & 0.2049 & 0.0237 \\
\hline 7 & 0.1094 & 0.0298 & 0.0237 \\
\hline 8 & 0.0781 & 0.0931 & 0.0394 \\
\hline 9 & 0.1015 & 0.2980 & 0.0237 \\
\hline 10 & 0.1172 & 0.2980 & 0.0237 \\
\hline 11 & 0.1015 & 0.0372 & 0.0315 \\
\hline 12 & 0.0156 & 0.2607 & 0.0158 \\
\hline
\end{tabular}

C. The Determination of the Ideal Solution and the Negative Ideal Solution

Let $x_{j}^{*}$ be the $j$ attribute value of the ideal solution of $x^{*}$, and $x_{j}^{0}$ is the $j$ attribute value of the negative ideal solution $x^{0}$, there are formulas (12) and (13).

$$
\begin{gathered}
x_{j}^{*}=\left\{\begin{array}{ll}
\max _{i} x_{i j} & j(\text { Benefit type attribute) } \\
\min _{i} x_{i j} & j(\text { Cost type attribute) }
\end{array} \quad j=1, \cdots, n\right. \\
x_{j}^{0}=\left\{\begin{array}{ll}
\max _{i} x_{i j} & j(\text { Benefit type attribute) } \\
\min _{i} x_{i j} & j(\text { Cost type attribute) }
\end{array} j=1, \cdots, n\right.
\end{gathered}
$$

By table 6 , formula(12)and formula(13), we can get the ideal solution and the negative ideal solution as follows.

$$
x^{*}=\left\{\begin{array}{lll}
0.1328 & 0.0037 & 0.0631
\end{array}\right\}
$$




$$
x^{0}=\left\{\begin{array}{lll}
0.0156 & 0.2980 & 0.0158
\end{array}\right\}
$$

D. Calculating the Distance of the Ideal Solution and the Negative Ideal Solution to the Weighted Attribute Value Respectively

Let $d_{i}^{*}$ be the distance between $x_{i}$ and the ideal solution, as shown in formula (14).

$$
d_{i}^{*}=\sqrt{\sum_{j=1}^{n}\left(x_{i j}-x_{j}^{*}\right)^{2}}, i=1, \cdots, m
$$

Let $d_{0}^{*}$ be the distance between $x_{i}$ and the negative ideal solution, as shown in formula (15).

$$
d_{i}^{0}=\sqrt{\sum_{j=1}^{n}\left(x_{i j}-x_{j}^{0}\right)^{2}}, i=1, \cdots, m
$$

Calculate $d_{i}^{*}$ and $d_{i}^{0}$ to determine the distance of $x^{*}$ and $x^{0}$ to the weighted multiple attribute values of the bottom events, as shown in table 7 , respectively.

\section{E. Calculating the Queue Indication Value}

$C_{i}^{*}$ is used to express the queuing indicator value, that is, the comprehensive evaluation index of the bottom event diagnosis, and its calculation method is shown in formula (16).

$$
C_{i}^{*}=d_{i}^{0} /\left(d_{i}^{0}+d_{i}^{*}\right), i=1, \cdots, m
$$

The queue indication value can be calculated with the formula(16) and the distances $d_{i}^{*}$ and $d_{i}^{0}$, as shown in table 6 .

TABLE VI. DISTANCE AND QUEUING INDICATOR VALUE CALCULATION TABLE

\begin{tabular}{|c|l|l|l|}
\hline Number & $d_{i}^{*}$ & $d_{i}^{0}$ & $C_{i}^{*}$ \\
\hline 1 & 0.0555 & 0.3045 & 0.84588 \\
\hline 2 & 0.0556 & 0.3009 & 0.84403 \\
\hline 3 & 0.0708 & 0.2567 & 0.78391 \\
\hline 4 & 0.1593 & 0.1709 & 0.51752 \\
\hline 5 & 0.1800 & 0.1506 & 0.45556 \\
\hline 6 & 0.2323 & 0.0938 & 0.28758 \\
\hline 7 & 0.0528 & 0.2842 & 0.84344 \\
\hline 8 & 0.1074 & 0.2155 & 0.66732 \\
\hline 9 & 0.2985 & 0.0863 & 0.22422 \\
\hline 10 & 0.2973 & 0.1018 & 0.25517 \\
\hline 11 & 0.0556 & 0.2750 & 0.83174 \\
\hline 12 & 0.2864 & 0.0372 & 0.11509 \\
\hline
\end{tabular}

According to the $C_{i}^{*}$ value, the optimal diagnosis sequence is determined from large to small, and the bottom event with large $C_{i}^{*}$ value is first diagnosed. It is known from table 7 that the optimal diagnosis sequence of the fault tree bottom events of the brake system is $S_{1}, S_{2}, S_{7}, S_{11}, S_{3}, S_{8}, S_{4}, S_{5}, S_{6}$, $S_{10}, S_{9}, S_{12}$.

It can be seen that in the process of fault diagnosis, besides taking into account the probability of the failure of the bottom event, the cost of diagnosis and the associated value should also be considered to determine the more reasonable diagnosis sequence of the fault tree bottom event, which can make the fault diagnosis and location process more rapid, effective and lower cost.

$$
d_{i}^{0}=\sqrt{\sum_{j=1}^{n}\left(x_{i j}-x_{j}^{0}\right)^{2}}, i=1, \cdots, m
$$

Calculate $d_{i}^{*}$ and $d_{i}^{0}$ to determine the distance of $x^{*}$ and $x^{0}$ to the weighted multiple attribute values of the bottom events, as shown in table 7 , respectively.

\section{CONCLUDING REMARKS}

On the basis of building a fault tree of chassis brake system for a launch vehicle, this paper carries out fault location for chassis braking system. Considering the probability of bottom event, diagnostic cost and associated value, the TOPSIS method is applied to determine the order of bottom event diagnosis. By comparing the traditional fault tree analysis method, this proposed method can identify the process of fault diagnosis more objectively, improve the accuracy of decision results and the efficiency of fault diagnosis, and enrich the fault tree analysis method, which has certain theoretical value.

\section{REFERENCES}

[1] ZHU Jizhou. The principle and application of fault tree[M]. Xian: Xian Jiaotong University Press, 1992: 25-36.

[2] LI Tianke, YU Shicai, FAN Hui, et al. Fault diagnosis equipment of missile launch vehicle control system[J]. Ordnance Industry Automation, 2015, 34(11): 33-37.

[3] CAO Ming, LIU Yabin. Application of fault tree analysis to seeker fault diagnosis[J]. Electronic Design Engineering, 2016, 24(11): 118-119,123.

[4] LI Shuying, WANG Peizhen, YANG Chun. Research and Application of Fault Diagnosis Based on BDD[J]. Journal of Electrical Engineering, 2017, 12(2): 38-42.

[5] ZHANG Yan, SHE Wei, LI Ping. Fault diagnosis model of electric power systems based on petri-nets and fault tree analysis[J]. Computer Measurement \& Control, 2015, 23(8): 2626- 2632.

[6] YAO Kairui, JIA Xuan, CAI Weifeng. Research of fault detection expert system of rocket launch vehicle electrical system based on fault Tree[J]. Industrial Control Computer, 2016, 29(2): 73-79.

[7] XIAO Guangyuan, SHEN Xiangjun, LI Zhigang. Fault tree analysis of launching canister based on fuzzy theory[J]. Ordnance Industry Automation, 2016, 35(11): 40-43.

[8] HE Jiazhou. Design and implementation of intelligent fault diagnosis method for a military radar[D]. Beijing: Tsinghua University, 2015.

[9] DONG Zewei, CHEN Wei, WANG Xudong, et al. Research of a certain armed helicopter weapon system fault diagnosis[J]. Fire Control \& Command Control, 2017, 42(1): 150-153.

[10] YAO Chengyu, DANG Zhen, CHEN Dongning, et al. Fault search strategy of hydraulic system based on T-S fuzzy fault tree analysis[J]. Journal of Yanshan University, 2011, 35(5): 407-412. 
[11] WANG Yongchang, ZHAO Jingyi, ZHANG Qisheng. FTA -Based fault diagnosis and search strategy for hydraulic system of rubber press[J]. China Mechanical Engineer -ing, 2002, 12(8): 874-879.

[12] SONG H, ZHANG H Y, CHAN C W. Fuzzy fault tree analysis based on T-S model with application to INS/GPS navigation system [J]. Soft Computing, 2009, 13(1): 31-40. 